\title{
WORK OF PERE MENAL ON NORMAL SUBGROUPS
}

\author{
F. A. ARimghaus and L. N. Vaserstein
}

Abstract

We describe subgroups of $G L_{2} A$ which are normalized by elementary matrices for rings $A$ satisfying the first stable range condition, Banach algebras $A$, von Veumann regular rings $A$, and other rings A.

Let $A$ be an associative ring with 1 and, for any natural number $n$, let $G L_{n} A$ be the group of all invertible $n$ by $n$ matrices with entries in $A$ (thus $G L_{1} A$ is the group of urits of $A$ ). Let $E_{n} A$ be the subgroup of $G L_{n} A$ generated by all elementary matrices $a^{i, 3}$, where $a \in A$ and $1 \leq i \neq j \leq n$.

For any subset $X$ of $A$, let $E_{n} X$ denote the subgroup of $G L_{n} A$ generated by all clementary matrices with entries in $X$, and let $E_{n}(A, X)$ denote the normal subgroup of $E_{n} A$ generated by $E_{n} X$. If $n \geq 3$ and $B$ is an ideal of $A$, then $E_{n}(A, B)$ is generated by elements of the form $a^{i, j} b^{j, i}(-a)^{i, j}$, where $a \in A, b \in B$, and $1 \leq i \neq j \leq n$.

Denote by $G_{n}(A, B)$ the inverse image of the center of $G L_{n}(A / B)$ under the canonical homomorphism $G L_{n} A \rightarrow G L_{n}(A / B)$; if $n \geq 2$, then $G_{n}(A, B)$ consists of all matrices of $G L_{n} A$ which, reduced modulo $B$, are scalar matrices $x I$ with $x$ in the center of $A / B$.

For various classes of rings $A$ (see $[3],[36]-[38]$ ) including all von Neumann rcgular rings, all Banach algebras, all commutative rings, and all stable range 1 rings, one has a complcte description of all subgroups $H$ of $G L_{n} A$ which are normalized by $E_{n} A$ for $n \geq 3$. Namely, for any subgroup $H$ of $G L_{n} A$ :

(a) If there exists an ideal $B$ of $A$ such that $E_{n}(A, B) \subset H \subset G_{n}(A, B)$, then $E_{n}(A, B)=\left[E_{n} A, E_{n} B\right]=\left[H, E_{n} A\right]=:\left[G_{n}(A, B), E_{n} A\right]$ and so $H$ is normalized by $E_{n} A$.

(b) If $H$ is normalized by $E_{n} A$, then there is a unique ideal $B$ of $A$ satisfying

$$
E_{n}(A, B) \subset H \subset G_{n}(A, B)
$$


Note that these cannot be extended to arbitrary rings $A$ when $n \geq 3$ (see [10]), nor are they true when $n=2$. In fact, the structure of $E_{2} A$ is intractable even for the integers $Z$ (see [9], [18], [27]-[32]). Since there are nonstandard normal subgroups of $E_{2} A$ when $A$ is a field of 2 or 3 elements (see [8]), the same is true of any ring $A$ with a residue field of 2 or 3 elements.

A ring $A$ is called von Neumann regular if for every $a \in A$ there is an $x \in A$ with $a x a=a$. By using $x a x$ instead of $x$, if necessary, we have $a x a=a$ and $x a x=x$.

Some partial positive results about the structure of $E_{2} A$ were known for von Neumann regular rings (see [37]), Banach algebras (see [38]), and rings with stable range 1 (see [3]), including all commutative rings $A$ with stable range 1 (see $[5],[20],[34],[43]$ ).

In particular, it was proved in [37] that for $A / \mathrm{rad} A$ von Neumann regular and $B$ an ideal of $A$, then $\left[E_{2} A, G_{2}(A, B)\right] \subset E_{2}(A, B)$. This inclusion implies that every subgroup $H$ of $G L_{2} A$ satisfying condition (a) with $n=2$ for some ideal $B$ is normalized by $E_{2} A$. However, these results excluded those normal subgroups of $E_{2} A$ which do not satisfy any ladder condition $E_{2}(A, B) \subset H \subset G_{2}(A, B)$ for any ideal $B$, for example when $A$ is the field of 2 or 3 elements and $H=\left[E_{2} A, E_{2} A\right]$. In [24], Menal and Vaserstein replaced $E_{2}(A, B)$ by $\left[E_{2} A, E_{2}(A, B)\right]$ to obtain the following complete description of normal subgroups of $E_{2} A$ for any von Neumann regular ring $A$.

Theorem 1. Let $A$ be a von Neumann regular ring and $H$ a subgroup of $G L_{2} A$ :

(a) If there is an ideal $B$ of $A$ with $\left[E_{2} A, E_{2} B\right] \subset H \subset G_{2}(A, B)$, then

$$
\left[H, E_{2} A\right] \subset E_{2} B=E_{2}(A, B)
$$

and

$$
\begin{aligned}
{\left[G_{2}(A, B),\left[E_{2} A, E_{2} A\right]\right] } & =\left[E_{2} A,\left[E_{2} A, H\right]\right]= \\
=\left[H \cap E_{2} A, E_{2} A\right] & =\left[E_{2} A, E_{2} B\right]=\left[E_{2} B, E_{2} B\right]
\end{aligned}
$$

$\subset H$. In particular, $H$ is normalized by $\left[E_{2} A, E_{2} A\right]$ and both $H \cap E_{2} A$ and $H E_{2} B$ are normalized by $E_{2} A$;

(b) If $\left[H, E_{2} A\right] \subset H$, then there is a unique ideal $B$ of $A$ such that

$$
\left[E_{2} A, E_{2} B\right] \subset H \subset G_{2}(A, B) \text {. }
$$

This gives the following classification result. 
Corollary. Let $A$ be a von Neumann regular ring. Then a subgroup $H$ of $E_{2} A$ is normal if and only if there is an ideal $B$ of $A$ such that $\left[E_{2} A, E_{2} B\right] \subset H \subset G_{2}(A, B)$.

In the case of a commutative regular ring $A$ this result was proved by Costa and Keller in [5]. Note that in this case $A$ has stable range 1 (see [11, cor. 4.5]), so $E_{2} A=S L_{2} A$. In general, the stable range of $A$ is not always 1 (see [23]), and it remains an open problem as to whether $A$ is always a $G E$-ring, i.e., $G L_{n} A$ is generated by clementary and diagonal matrices.

The uniqueness of the ideal $B$ in the theorem is easy to see. This is a consequence of the following fact which is true for an arbitrary ring $A$ : if $H$ is a subgroup of $G L_{2} A$ such that $\left[E_{2} A, E_{2} B\right] \subset H \subset G_{2}(A, B)$ for some ideal $B$ of $A$ then $B=\left\{x \in A: x^{1,2} x^{2,1} \in H\right\}$. To verify this fact, observe that $x^{1,2} x^{2,1}=\left[x^{1,2}, 1^{1,2}(-1)^{2,1} 1^{1,2}\right] \in\left[E_{2} B, E_{2} A\right] \subset H$ for any $x \in B$. Finally, since both off-diagonal entries of the matrix are $x$, the inclusion $x^{1,2} x^{2,1} \in G_{2}(A, B)$ implies that $x \in B$.

In general, the intersection $H \cap E_{2} A$ in the theorem cannot bo replaced by $H$ because $\left[E_{2} B, E_{2} A\right] \neq\left[H, E_{2} A\right]$ when $I I=G_{2}(A, B)$ and $A=B$ is the field of three elements. However, when $E_{2} A$ is perfect (i.e., $E_{2} A=$ $\left.\left[E_{2} A, E_{2} A\right]\right)$, the theorem implies that $\left[E_{2} A, E_{2} B\right]=\left[G_{2}(A, B), E_{2} A\right]$. In this case, a subgroup $H$ of $G L_{2} A$ is normalized by $E_{2} A$ whenever $\left[E_{2} A, E_{2} B\right] \subset H \subset G_{2}(A, B)$ for an idcal $B$ of $A$.

In the same paper, Menal and Vaserstein also applied these techniques to Banach algebras. However, it was shown in $[39$, section 4$]$ that there are normal subgroups of $E_{2} A$ corresponding to any quasi-ideal $Y$ of $A$ which do not satisfy any ladder condition $\left[E_{2} A, E_{2} B\right] \subset H \subset G_{2}(A, B)$ for an ideal $B$ of $A$ if $Y$ is not itself an ideal, for example, $E_{2}(A, Y)$ (see [33, prop. 4.2]).

Recall that a quasi-ideal of $A$ is an additive subgroup $Y$ of $A$ such that aya $\in Y$ and $y a y \in Y$ for any $y \in Y$ and $a \in A$. Note that in many cases, every quasi-idcal is an ideal, for example, when $2 A=A$ and $A$ is commutative $[39$, lemma 4.1$]$, but that this is not true in gencral. In fact, if $2 A \neq A$, then quasi-ideals appear even in commutative local rings (see [1]).

The previous results did not use quasi-ideals because every quasi-ideal of a von Neumann regular ring is an ideal. This is also the case for every Banach algebra which is simple or commutative (see [38], [39]), but is not true for an arbitrary Banach algebra. However, the exterior (Grassman) algebra on a 2-dimensional real vector space is an cxample of a 4-dimensional algebra with a quasi-ideal that is not an ideal.

Let $C_{2}(A, Y)$ be the set of all $g \in G L_{2} A$ with $\left[g, E_{2} A\right] \subset E_{2}(A, Y)$. 
Clearly $C_{2}(A, B) \subset G_{2}(A, B)$ for any ring $A$ and any ideal $B$ of $A$; the equality $C_{2}(A, B)=G_{2}(A, B)$ is equivalent to the inclusion $\left[E_{2} A, G_{2}(A, B)\right] \subset E_{2}(A, B)$. Menal and Vascrstein then proved the following description of normal subgroups.

Theorem 2. Suppose that $2 A=A$ and that for any a $\in A$ there is a finite sequence $x_{1}, x_{2}, \ldots, x_{n}$ in $A$ with $x_{1}+x_{2}+\ldots+x_{n}=1$ and $1-a x_{i} \in G L_{1} A$ for all $i$. Let $H$ be a subgroup of $G L_{2} A$.

(a) If there is a quasi-ideal $Y$ of $A$ with $E_{2}(A, Y) \subset H \subset C_{2}(A, Y)$ then $E_{2}(A, Y)=\left[E_{2} A, E_{2} Y\right]=\left[E_{2} A, E_{2}(A, Y)\right]=\left[E_{2} A, H\right]=$ $\left[E_{2} A, C_{2}(A, Y)\right] \subset H$ and so $H$ is normalized by $E_{2} A$. Furthermore, when $Y$ is an ideal, $C_{2}(A, Y)=G_{2}(A, Y)$;

(b) If $H$ is normalized by $E_{2} A$, then there is a unique quasi-ideal $Y$ of $A$ such that $E_{2}(A, Y) \subset H \subset C_{2}(A, Y)$.

This leads to the following classification result.

Corollary. Suppose that $2 A=A$ and that for any $a \in A$ there is a finite sequence $x_{1}, x_{2}, \ldots, x_{n}$ in $A$ with $x_{1}+x_{2}+\ldots+x_{n}=1$ and $1-a x_{i} \in G L_{1} A$ for all $i$. A subgroup $H$ of $E_{2} A$ is normal if and only if there is a quasi-ideal $Y$ of $A$ such that $E_{2}(A, Y) \subset H \subset C_{2}(A, Y)$.

Menal and Vaserstein proved some parts of the theorem under weaker hypotheses on the ring $A$. It should also be noted that under slightly different hypotheses on the ring $A$, the theorem was proved in [39] in the case when every quasi-ideal of $A$ is an idcal.

The hypotheses of Theorcm 2 are satisfied not only by Banach algebras but also by many other rings, for example, the ring of all bounded smooth functions on any smooth manifold. In fact, these hypotheses are satisfied by any connected topological ring $A$ with $G L_{1} A$ open in $A$.

Theorem 2 can also be extended to other classes of rings. We call $A$ semilocal if $A / \mathrm{rad} A$ is a (not necessarily finite) direct product of matrix rings over division rings - for example, any Artinian ring is sernilocal. Menal and Vaserstein showed in [24] that the semilocal rings satisfying the hypotheses of Theorem 2 are precisely those rings $A$ with $2 A=A$ and which contain no factor ring isomorphic to a matrix ring $M_{n}(\mathrm{Z} / 3 \mathrm{Z})$, which extends Theorem 2 to any subgroup $H$ of $G L_{2} A$ in the following manner.

Corollary. Let $2 A=A$ be a semilocal ring without factor rings isomorphic to a matrix ring $M_{n}(\mathbf{Z} / 3 \mathbf{Z})$. Then the conclusions of Theorem 2 hold for any subgroup $\mathrm{H}$ of $G L_{2} A$. 
For a commutative local ring, the corollary was proved in ([1] ; [13], [15], [16], [19], [21], [33]); results for commutative semilocal rings can be found in $([2],[7])$. Kolotilina and Vavilov showed in [14] that for any normal subgroup $H$ of $G L_{2} A$ there is a unique ideal $B$ of $A$ such that $E_{2}(A, B) \subset H \subset G_{2}(A, B)$, provided that $A$ is a semilocal ring satisfying two conditions. First, the center of $A$ must contain a unit $\varepsilon$ such that $1-\varepsilon$ is also a unit; second, that $A$ has neither factor rings which are division algebras with centers of cardinality less than 5 nor factor rings which are isomorphic to $M_{2}(\mathbf{Z} / 2 Z)$.

The use of quasi-ideals opens up a wider range of rings. Many authors had some treatment of commutative local rings, but the results that Menal and Vaserstein obtained in [24] included noncommutative local rings $A$ for which $2 A=A$. In [26], they extended their results to local rings $A$ such that $A / \operatorname{rad} A$ contained at least 4 elements.

We call a ring $A$ local if $A / \operatorname{rad} A$ is a division ring, where $\operatorname{rad} A$ denotes the Jacobson radical of $A$. Note that in a local ring $A$, if $Y$ is a quasiideal of $A$ and $Y \neq A$, then $Y \subset \operatorname{rad} A$, since $A=y A y \subset Y$ if $Y$ contains a unit $y$.

Let $A$ be the exterior (Grassman) algebra on a vector space $V$ over a field $F$ with $\operatorname{dim}_{F} V \geq 2$ and let $Y$ be the $F$-subspace of $A$ spanned by the monomials of odd degree. Then $A$ is a local ring with $A / \mathrm{rad} A=F$ and $Y$ is a quasi-ideal which is not an ideal. If $2 F=F$, then $2 A=A$ and $A$ is not commutative. If $2 F=0$, then $2 A=0$ and $A$ is commutative.

For any quasi-ideal $Y \neq A$ of an arbitrary ring $A$ denote by $T_{2}(A, Y)$ the set of all elements of the form $x^{2,1} \operatorname{diag}(u, v) y^{1,2}$, where $x, y \in Y$, $u, v \in G L_{1} A$, and $v^{-1} a u-a, u a v^{-1}-a \in Y$ for all $a \in A$. Set $T_{2}(A, A)=$ $G L_{2} A$. When $A$ is local, it is clear that $T_{2}(A, B)=G_{2}(A, B)$ for every ideal $B$ of $A$. Note that for any ring $A, G_{2}(A, 0)$ is the conter of $G L_{2} A=$ $G_{2}(A, A)$ which consists of scalar matrices over the center of the ring $A$. Moreover, $G_{2}(A, 0)$ is the centralizer of $E_{2} A$ in $G L_{2} A$.

Then Menal and Vaserstein proved the following result.

Theorem 3. Suppose $A$ is a local ring such that $A / r a d ~ A$ has at least 4 elements. Then:

(a) $E_{2}(A, Y)=\left[E_{2} A, E_{2} Y\right]=\left[E_{2} A, E_{2}(A, Y)\right]=\left[E_{2} A, T_{2}(A, Y)\right]$ for any quasi-ideal $Y$ of $A$;

(b) If $H$ is a subgroup of $G L_{2} A$ which is normalized by $E_{2} A$, then there exists a unique quasi-ideal $Y$ of $A$ such that $E_{2}(A, Y) \subset$ $H \subset T_{2}(A, Y)$.

This result uses the following lemma, which follows from $T_{2}(A, Y)$ bcing a subgroup of $G L_{2} A$ invariant under conjugation by $E_{2} A$. 
Lemma. Let $A$ be a local ring, $Y$ a quasi-ideal of $A$. Then $T_{2}(A, Y)$ is a subgroup of $G L_{2} A$ and $\left[E_{2} A, T_{2}(A, Y)\right] \subset E_{2}(A, Y) \subset T_{2}(A, Y)$. Therefore $\left[H, E_{2} A\right] \subset H$ for any subgroup $H$ of $G L_{2} A$ such thot $E_{2}(A, Y) \subset H \subset T_{2}(A, Y)$ for some quasi-ideal $Y$ of $A$.

The unique quasi-ideal in part (b) of Theorem 3 is in fact the level of $H, L(H)=\left\{a \in A: a^{1,2} \in H\right\}$ which plays an important role in most classification theorems. The uniqueness of $Y$ is easy to sce, because $Y \subset L(H)$ whenever $E_{2}(A, Y) \subset H$, and $L(H) \subset Y$ whenever $H \subset T_{2}(A, Y)$. This is true for an arbitrary ring $A$ and a subset $Y$; however in general it is not true that $E_{2}(A, Y) \subset T_{2}(A, Y)$.

If card $(A / \mathrm{rad} A)<4$, then $E_{2} A$ maps onto the group $E_{2} F=S L_{2} F$ where $F=A / \mathrm{rad} A$ is a field, and since $E_{2} F$ is not perfect (see [8]), $E_{2} A$ is not perfect.

Furthermore, in this case, $H=\left[E_{2} A, E_{2} A\right]$ is a normal subgroup of $E_{2} A$ such that no quasi-ideal $Y$ of $A$ exists with $E_{2}(A, Y) \subset H \subset$ $T_{2}(A, Y)$. In fact, if $A$ is the field of 2 elements, then $H$ is a non-central normal subgroup of $G L_{2} A$ containing no nontrivial elementary matrices.

In the case of a commutative local ring $A$, the theorem is essentially due to Abe (see [1]). This case was also treated in ([13], [15], [16], [19], [21], [33]). When $2 A=A$, the theorem is contained in Menal and Vaserstein (see [24]). The main difficulties in proving the theorem lic in the case when $A / \mathrm{rad} A$ is a small field of characteristic 2 .

It was shown in [42] that for any local ring $A$ with $A / \mathrm{rad} A$ having at least 3 elements and any normal subgroup $H$ of $G L_{2} A$ there is a unique ideal $B$ of $A$ such that $E_{2}(A, B) \subset H \subset G_{2}(A, B)$. Under the additional condition that the center of $A / \mathrm{rad} A$ has at least 7 elements, this was proved previously by Kolotilina and Vavilov in [14]. However, it is not truc in general that every subgroup $H$ of $G L_{2} A$ such that $E_{2}(A, B) C$ $H \subset G_{2}(A, B)$ for an ideal $B$ of $A$ is normal (see [42]). By the lemma, such a subgroup $H$ is always normalized by $E_{2} A$.

In [25], Menal and Vaserstein used these methods on stable range one rings. In this paper they generalized to noncommutative rings the description of $E_{2} A$-normalized subgroups given by Costa and Keller in [5]. The quasi-ideals which appear in the results of Menal and Vaserstein do not appear in many previous results as every quasi-ideal is an ideal in the commutative case with $2 A=A$.

A ring $A$ satisfies the first Bass stable range condition if for any $a, b \in A$ with $a A+b A=A$, there is an $r \in A$ such that $(a+b r) A=A$ (sce $[35]$ ). Equivalently, this means that there is an $r$ such that $a+b r$ is a unit. We denote this by $\operatorname{sr}(A) \leq 1$. A result of Kaplansky [40] says that when $\operatorname{sr}(A) \leq 1$ every one-sided unit in $A$ is a unit. 
In [12], Goodearl and Menal proved the stable range one condition for many classes of rings and algebras. They used a strong form of stable range one, unit 1-stable range (replace the $r$ above by a unit $u$ to obtain the definition), which followed from the following condition on a ring $A$ : given any $x, y \in A$, there is a unit $u \in A$ such that $x-u$ and $y-u^{-1}$ are both units. Verification of this condition yields stable range one in the following cases: (1) any algebra over an uncountable field, in which all elements are zero-divisors or units and there are no uncountable direct sums of nonzero one-sided ideals; (2) any algebra over an uncountable ficld, in which there are only countably many primitive factor rings, all of which arc Artinian; (3) the endomorphism ring of any noetherian module over an algebra as in (2); (4) any algebraic algebra over an infinite field; (5) any integral algebra over a commutative ring which modulo its (Jacobson) radical is algebraic over an infinite field; (6) any von Neumann regular algebra over an uncountable field, which has a rank function. They also use other techniques to prove stable range 1 for other rings, including finite Rickart $C^{*}$-algebras and certain strongly $\pi$-regular rings. For more examples of stable range one rings, see [3], [6], [35], [40], [41].

When $\operatorname{sr}(A) \leq 1$, more is known about the structure of $G L_{n} A$. In [3], Bass showed

$$
\left[E_{n} A, E_{n} B\right]=\left[E_{n} A, G_{n}(A, B)\right]=\left[G L_{n} A, G L_{n} B\right]=E_{n}(A, B)
$$

for any idcal $B$ of $A$ and any $n \geq 3$, where the principal congruence subgroup $G L_{\pi i} B$ is defined as the kerncl of the homomorphism $G L_{n} A \rightarrow$ $E_{n}(A / B)$. Generally, $\left[G L_{n} A, G_{n}(A, B)\right] \neq E_{n}(A, B)$ even for local rings $A$. Moreover, $G_{n}(A, B) / G L_{n} B=G_{2}(A, B) / G L_{2} B$ for $n \geq 2$, hence this group does not depend on $n$, and it is an abelian group isomorphic to the group of units of the center of the ring $A / B$.

The group $G L_{n} B / E_{n}(A, B)=K_{1}(A, B)=G L_{1} B / W(A, B)$ is also an abelian group which does not depend on $n$ for $n \geq 2$, where $W(A, B)$ is the subgroup of $G L_{1} A$ generated by the elements of the form $(a+c+a b c)(a+c+c b a)^{-1}$ with $a \in B, b \in A, c \in 1+B$ (see [17], [22]). So the group $G_{n}(A, B) / E_{\pi}(A, B)$, which classifics all subgroups $H$ of $G L_{n} A$ normalized by $E_{n} A$ and with the same "level" $B$, is two-step nilpotent. When $A$ is commutative, $E_{n}(A, B)=S L_{n} B$ for all ideals $B$ of $A, G_{n}(A, B) / G L_{n} B=G 1(A, B) / G L_{1} B=G L_{1}(A / B)$, and $G L_{n} B / E_{n}(A, B)=K_{1}(A, B)=G L_{1} B$.

The next theorem [25] gives more information about the structure of $E_{2}(A, Y)$ and $C_{2}(A, Y)$ for rings $A$ with $\operatorname{sr}(A) \leq 1$ and quasi-ideals $Y$ of $A$. Part (b) generalizes to quasi-ideals a result of [15] describing the 
kernel of the Whitehead deterninant $G L_{1} B \rightarrow K_{1}(A, B)$ for ideals $B$ (the case $B=A$ had been done in [18]).

Theorem 4. Let $A$ be an associative ring with $\operatorname{sr}(A) \leq 1$. Then for any quasi-ideal $Y$ of $A$ :

(a) the group $E_{2}(A, Y)$ is generated by elements of the forms $a^{1,2} y^{2,1}(-a)^{1,2}$ and $a^{2,1} y^{1,2}(-a)^{2,1}$ with $a \in A$ and $y \in Y$;

(b) the group $E_{2}(A, Y)$ consists of all elements of the form $x^{1,2} y^{2,1} z^{1,2} d$, where $x, y, z \in Y$, and $d$ is a diagonal matrix which is a product of diagonal matrices of the form diag $(a+c+a b c,(a+$ $c+c b a)^{-1}$ ) with $a, b, c \in A, a+c+c b a \in G L_{1} A$ and either $a-1, c \in Y$ or $a, c-1 \in Y$;

(c) when $Y$ is an ideal of $A, C_{2}(A, Y)=G_{2}(A, Y)$ and this group consists of all elements of the form $x^{1,2} y^{2,1} z^{1,2} d$ with $x, y, z \in Y$ and $a$ diagonal matrix $d=\operatorname{diag}(u, v)$ with $u, v \in G L_{1} A$ such that $v^{-1} a u-a, u a v^{-1}-a \in Y$ for all $a \in A$.

Under the additional condition $2 A=A$, Menal and Vaserstein proved the sccond conclusion of Theorem $4(\mathrm{c})$ for any quasi-ideal $Y$ of $A$ :

Theorem 5. Let $A$ be an associative ring with 1 such that $\operatorname{sr}(A) \leq$ 1 and $2 A=A$. Then for any quasi-ideal $Y$ of $A$, the group $C_{2}(A, Y)$ consists of all elements of the form $x^{1,2} y^{2,1} z^{1,2} d$ with $x, y, z \in Y$ and a diagonal matrix $d=\operatorname{diag}(u, v)$ with $u, v \in G L_{1} A$ such thot $v^{-1} a u-$ $a, u a v^{-1}-a \in Y$ for all $a \in A$.

In this case, Menal and Vaserstein obtained the following result.

Theorem 6. Let $A$ be an associative ring with 1 such that $2 A=A$ and $\operatorname{sr}(A) \leq 1$. Then a subgroup $H$ of $E_{2} A$ is normal if and only if $\left[E_{2} A, E_{2}(A, Y)\right] \subset H \subset C_{2}(A, Y)$ for some quasi-ideal $Y$ of $A$.

This classification result follows from:

Theorem 7. Let $A$ be an associative ring with 1 such that $2 A=A$ and $\operatorname{sr}(A) \leq 1$.

(a) If $H$ is a subgroup of $G L_{2} A$ normalized by $E_{2} A$, then there is a unique quosi-ideal $Y$ of $A$ such that $\left[E_{2} A, E_{2}(A, Y)\right] \subset H \subset$ $C_{2}(A, Y)$;

(b) If $Y$ is a quasi-ideal of $A$, then $\left\{E_{2} A, E_{2} Y\right\}=\left[E_{2} A, C_{2}(A, Y) \cap\right.$ $\left.E_{2} A\right]$. 
For a stable range 1 ring $A$ and $n \geq 3,\left(G L_{n} A\right)_{a b}$ : the abelianization of the linear group $G L_{n} A$ is woll-known. Specifically, it is $K_{1} A$ which is isomorphic to $G L_{1} A / W(A)$, which is described above. In [4], Berrick and Menal computed the abclianization of $G L_{2} A$, showing that $K_{1} A$ is a direct summand of $\left(G L_{2} A\right)_{a b}$ in the following result.

Theorem 8. If $I$ is an ideal of $A$ with stable range 1 , then every element $g \in G L_{2} I$ can be written in the form $g=\operatorname{diag}(u, v) a^{2,1} b^{1,2} c^{2,1}$, where $u, v \in G L_{2} I$ and $a, b, c \in I$. Moreover, there is a group isomorphism of $\left(G L_{2} I\right)_{a b}$ with $\left(I / I^{2}\right)^{3} \oplus I^{2} / L \oplus G L_{1} I / W_{1}(I, I)$, where $L$ is the ideal of $I$ generated by $\left(x^{2}-x\right) I$ and $I\left(x^{2}-x\right)$ for all $x \in I$.

In [42], the problem of developing a "sandwich theorem" for normal subgroups of $G L_{2} A$ similar to the one for $G L_{n} A$ with $n \geq 3$ was explored. In this paper, results prcviously discussed were generalized to rings $A$ which satisfy one of threc conditions; either $A$ is a stable range 1 ring, or $A / \mathrm{rad} A$ is von Neumann regular, or for every $a \in A$ there is a finite sequence $x_{1}, x_{2}, \ldots, x_{n}$ in $A$ such that $x_{1}+x_{2}+\ldots+x_{n}=1$ and $1-a x_{i} \in G L_{1} A$ for all $i$. In this case, the following theorem was proved.

Theorem 9. Suppose $A$ is a ring satisfying one of the above three conditions such that no proper one-sided ideal of $A$ contains all $u-1$, where $u \in G L_{1} A$, and every element of $A$ is a sum of units. Then for every subgroup $H$ of $G L_{2} A$ which is normalized by $G E_{2} A$ there is a unique ideal $B$ of $A$ such that $\left[E_{2} A, E_{2} B\right] \subset H \subset G_{2}(A, B)$.

Here $G E_{2} A$ is the subgroup of $G L_{2} A$ generated by all its diagonal and elementary matrices. If $A$ is a stable range 1 ring, then $G E_{2} A=G L_{2} A$. When no proper one-sided ideal of $A$ contains all $u-1, E_{2}(A, B) \subset$ $\left[E_{2} B, G E_{2} A\right]$ for every ideal $B$ of $A$.

While the converse to this theorem is not always true, the same paper contains the following modification.

Theorem 10. Suppose A satisfies one of the above three conditions. Then

$$
\left[G_{2}(A, B),\left[E_{2} A, E_{2} A\right]\right] \subset E_{2}(A, B)
$$

for any ideal $B$ of $A$. Therefore, if $H$ is a subgroup of $G L_{2} A$ such that $E_{2}(A, B) \subset H \subset G_{2}(A, B)$ for an ideal $B$ of $A$, then $\left[H,\left[E_{2} A, E_{2} A\right]\right] \subset$ $E_{2}(A, B)$, hence $H$ is normalized by $\left[E_{2} A, E_{2} A\right]$. 


\section{References}

1. E. ABE, Chevalley groups over local rings, Tohôku Math. J. 21 (1969), 474-494.

2. J. B. AN AND X. P. TANG, The structure of symplectic groups over semilocal rings, Acta Math. Sinica (N.S.) 1, no. 1 (1985), 1-15.

3. H. BAss, $K$-theory and stable algcbra, Publ. Math. IHES 22 (1964), 5-60.

4. J. A. BERRICK AND P. MENAL, On the abelianization of $G L_{2}$ for rings with stable range 1 , Preprint.

5. D. L. Costa AND G. E. Keller, On the normal subgroups of $S L(2, A)$, J. Pure Appl. Algebra 53 (1988), 201-227.

6. D. L. CostA, Zero-dimensionality and the $G E_{2}$ of polynomial rings, J. Pure Appl. Algebra 50 (1988), 223-229.

7. C. N. Chang, The structure of the symplectic group over semi-local domains, J. Algebra 35 (1975), 457-476.

8. L. E. Dickson, Theory of linear groups in arbitrary field, Trans. Amer. Moth. Soc. 2 (1901), 363 394.

9. B. Fine and M. Newman, The normal subgroup structure of the Picard group, Trans. Amer. Math. Soc. 302, no. 2 (1987), 769-786.

10. V. N. Gerasimov, The unit group of free products, Mat. Sb. 134, no. 1 (1987), 42-65.

11. K. R. GOODEARI, "Von Neumann regulor rings," Pitman, London, 1979.

12. K. R. Goodearl and P. Menal, Stable range one for rings with many units, J. Pure Appl. Algebra 54 (1988), 261-287.

13. W. KingenbeRG, Lineare Gruppenüber lokalen Ringen, Amer. $J$. Math. 83 (1961), 137. 153.

14. L. YU. KOlOtiliNa AND N. A. Vavilov, Normal structure of the full linear group over semilocal rings, J. Sov. Moth. 19, no. 1 (1982), 998.999.

15. N. H. J. LACRoIX, Two-dimensional linear groups over local rings, Can. J. Math. 21 (1969), 106-135.

16. N. H. J. Lacroix and C. Levesque, Sur les sous-groupes normaux de $S L_{2}$ sur un anneau local, Can. Math. Bull. 26, no. 2 (1983), 209-219.

17. A. W. Magurn and L. N. Vaserstein, Pre-stabilization for $K_{1}$ of Banach algebras, Linear Algebra 95 (1987), 69-96. 
18. A. W. MASON, Anomalous normal subgroups of $S L_{2} K[x]$, Quart. J. Math. 36, no. $143(1985), 345-358$.

19. A. W. MASON, On $G L_{2}$ of a local ring in which 2 is not a unit, Can. Math. Bull. 30, no. 2 (1987), 165-176.

20. B. R. MCDonald, $G L_{2}$ of rings with many units, Comm. Algebro 8, no. $9(1980), 869-888$.

21. B. R. MCDonalo, "Geometric algebra over local rings," New York-Basel, Marcel Dekker, 1976.

22. P. MEnal and J. Moncasi, $K_{1}$ of von Neumann regular rings, $J$. Pure Appl. Algebra 33 (1984), 295-312.

23. P. Menal And J. Moncasi, On regular rings with stable range 2, J. Pure Appl. Algebra 24 (1982), 25-40.

24. P. Menal and L. N. Vaserstein, On subgroups of $G L_{2}$ over Banach algebras and von Neumann regular rings which are normalized by elementary matrices, J. Algebra 138, no. 1 (1991), 99- 120.

25. P. MENAL AND L. N. VAserstein, On the structure of $G L_{2}$ over stable range one rings, J. Pure Appl. Algebra 64 (1990), 149-162.

26. P. MENAL AND L. N. VASERSTEIN, On normal subgroups of $G L_{2}$ over non-commutative local rings which are normalized by elementary matrices, Math. Ann. 285 (1989), $221-231$.

27. M. NEWMAN, A complete description of normal subgroups of genus one of the modular group, Amer. J. Math. 86 (1964), 17-24.

28. M. NEWMAN, Free subgroups and normal subgroups of the modular group, Mlinois Math. J. 8 (1964), 262-265.

29. I. REINER, Normal subgroups of the unimodular group, Illinois Math. J. 2 (1958), 142-144.

30. I. Reiner, Subgroups of the unimodular group, Proc. Amer. Math. Soc. 12 (1961), 173-174.

31. J. P. SERRE, Le problèmc des groupes de congruences pour $S L_{2}$, Ann. of Math. 92 (1970), 489-527.

32. A. A. Sustin, On a theorem of Cohn, J. Sov. Moth. 17, no. 2 (1981), 1801-1803.

33. S. TAzHETdinov, Subnormal structure of two-dimensional linear groups over local rings, Alg. Logic 22, no. 6 (1983), 707-713.

34. S. TAZHETDiNov, Subnormal structure of two-dimensional linear groups over rings that are close to fields, Alg. Logic 24, no. 4 (1985), 414-425.

35. L. N. VASERSTEIN, $K_{1}$-theory and the congruence subgroup problem, Math. Notes 5 (1969), 141-148. 
36. L. N. VASERStein, On normal subgroups of $G L_{n}$ over a ring, in Lecture Notes in Math 854, Springer-Verlag, New York/Berlin.

37. L. N. VASERSTEIN, Normal subgroups of the general linear groups over von Neumann regular rings, Proc. Amer. Math. Soc. 96, no. 2 (1986), 209-214.

38. L. N. VASfrStein, Normal subgroups of the general linear groups over Banach algebras, J. Pure Appl. Algebra 41 (1986), 99-112.

39. I. N. VASERSTEIN, Subnormal structure of the general linear groups over Banach algebras, J. Pure Appl. Algebra 52 (1988), 187-195.

40. L. N. Vaserstein, Bass's first stable range condition, J. Pure Appl. Algebra 34 (1984), 319-330.

41. L. N. VAserstein, An answer to the question of M. Newman on matrix completion, Proc. Amer. Math. Soc. 97 (1986), 189-196.

42. L. N. VASERSTEIN, On normal subgroups of $G L_{2}$ over rings with many units, Compositio Math. 74 (1990), $157 \cdot 164$.

43. I. Q. WANG AND Y. Z. Zhanc, $G L_{2}$ over full rings, Chinese Ann. Math. ser. B 8, no. 4 (1987), 434-439.

\author{
Department of Mathernatics \\ Penn State University \\ University Park \\ PA 16802 U.S.A.
}

Primera versió rebuda el 2 d'Octubre de 1991, darrera versió rebuda el 4 de Març de 1992 